UNIO - EU Law Journal. Vol. 5, No. 2, July 2019, pp 34-42.

®2019 Centre of Studies in European Union Law

School of Law - University of Minho

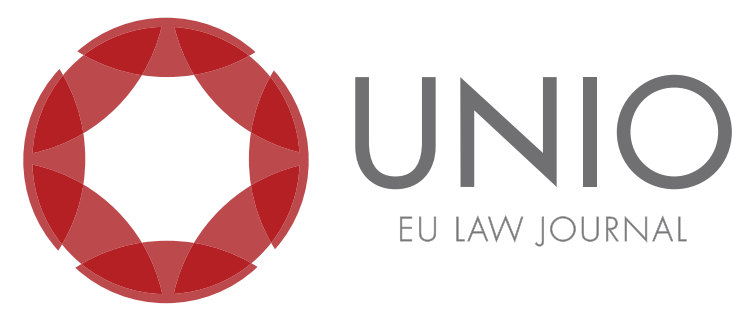

\title{
Directive on certain aspects concerning contracts for the supply of digital content and digital services \& the EU data protection legal framework: are worlds colliding?
}

\author{
Maria de Almeida Alves*
}

ABSTRACT: This Paper will address the interplay between the Directive on certain aspects concerning contracts for the supply of digital content and digital services and the current $E U$ data protection framework, namely the General Data Protection Regulation. Albeit the Directive has the aim of protecting consumers, has it gone too far and made a crack in the data protection EU legal framework? Can personal data be treated as a commodity or is its scope as a counter-performance subject to a particular interpretation? I shall analyze these questions in light of the European Data Protection Supervisor's Opinion 4/2017 and the European Data Protection Board's Guidelines 2/2019.

KEYWORDS: personal data - digital content and services - counter-performance - legal basis - harmonization.

* Lawyer at Vieira de Almeida \& Associados; Master in Literary Theory with a thesis entitled "Moral luck and the legal concept of attempt" at the University of Lisbon. 


\section{Introduction}

On the $15^{\text {th }}$ of April, the European Union adopted a package comprising a Directive on contracts for the supply of digital content and services ${ }^{1}$ ("DCD") and a Directive on contracts for the sale of goods ("SGD"). For the purpose of the present paper, I will solely analyze the DCD.

The DCD lays down common rules on certain requirements concerning contracts between traders and consumers for the supply of digital content and digital services. ${ }^{3} \mathrm{In}$ particular, the DCD lays down rules on the conformity of digital content and services and remedies in the event of a lack of conformity or failure to supply and modification of such content and service.

The DCD regulates the supply of digital content and covers (a) data produced and supplied in digital form (e.g. music and online video), (b) services allowing for the creation and processing of data in digital form (e.g. cloud), (c) services allowing for the share of data (e.g. YouTube and Facebook) as well as a durable medium used exclusively as a carrier of digital content (e.g. DVDs). ${ }^{4}$

For the purpose of this paper, the most significant innovation of the DCD lies in its scope of application, as the DCD applies not only where a consumer pays a price but also when he provides personal data as counter-performance. The question that arises is whether such a perspective - personal data as counter-performance - can be harmonized with the data protection legislation, namely the General Data Protection Regulation. ${ }^{5 / 6}$ Furthermore, how has the European Data Protection Supervisor ("EDPS") and the European Data Protection Board ("EDPB") construed such a perspective? Can the EDPB's Guidelines 2/20197 ("Guidelines") help with the task

\footnotetext{
${ }^{1}$ Directive 2019/770 of the European Parliament and of the Council of 20 May 2019 on certain aspects concerning contracts for the supply of digital content and digital services, OJ L 136, 22.5.2019, 1-27, available on: https:// eur-lex.europa.eu/legal-content/EN/TXT/?uri=CELEX:32019L0770.

${ }^{2}$ Directive 2019/771 of the European Parliament and of the Council of 20 May 2019 on certain aspects concerning contracts for the sale of goods, amending Regulation (EU) 2017/2394 and Directive 2009/22/EC, and repealing Directive 1999/44/EC, OJ L 136, 22.5.2019, 28-50, available on: https:/ / eur-lex.europa.eu/legal-content/EN/TXT/?uri=CELEX:32019L0771.

${ }^{3}$ See recital 86 of the DCD: "Since the objectives of this Directive, namely to contribute to the functioning of the internal market by tackling in a consistent manner contract law related obstacles for the supply of digital content or digital services while preventing legal fragmentation, cannot be sufficiently achieved by the Member States but can rather, by reasons of ensuring the overall coherence of the national laws through harmonised contract law rules which would also facilitate coordinated enforcement actions, be better achieved at Union level, the Union may adopt measures, in accordance with the principle of subsidiarity as set out in Article 5 of the Treaty on European Union. In accordance with the principle of proportionality, as set out in that Article, this Directive does not go beyond what is necessary in order to achieve those objectives."

${ }^{4}$ Over the top communication services (OT'Ts) and bundle contracts are included within the scope of the DCD.

${ }^{5}$ Regulation No. 2016/679 of the European Parliament and of the Council of 27 April 2016 on the protection of individuals with regard to the processing of personal data and on the free movement of such data and repealing Directive 95/46 on the protection of individuals with regard to the processing of personal data and on the free movement of such data (General Regulation on Data Protection GDPR), OJ L 119, 4.5.2016, 1-88, available on: https://eur-lex.europa.eu/legal-content/EN/TXT/ $\mathrm{PDF} /$ ?uri=CELEX:32016R0679\&from $=\mathrm{EN}$.

${ }^{6}$ Note that the GDPR applies to the processing of personal data of data subjects who are in the EU by a controller or processor not established in the Union, where the processing activities are related to "the offering of goods or services, irrespective of whether a payment of the data subject is required, to such data subjects in the Union". As such, the scope of the GDPR is not made dependent on the existence of any payment of counter-performance of any kind.
}

${ }^{7}$ EDPB's Guidelines on the processing of personal data under Article 6(1)(b) GDPR in the context of 
of unveiling what the practical reach of the DCD's scope is once read in line with the GDPR?

\section{Personal data as counter-performance in the DCD}

It is important to understand exactly what results from the interplay between the DCD and the EU data protection framework. Let me start by highlighting that the DCD does not have a specific definition of personal data; it has a remission to Article 4 of the GDPR. As such, we are presented with a very broad definition, which makes it likely that most data provided by a consumer to a provider of digital content or services will be considered as personal data. ${ }^{8}$

Concerning data as counter-performance, the directive applies "where the trader supplies or undertakes to supply digital content or a digital service to the consumer and the consumer provides or undertakes to provide personal data to the trader". ${ }^{9}$ Exceptions lie "where the personal data provided by the consumer is exclusively processed by the trader for supplying of the digital content or digital service [...] or for the trader to comply with legal requirements to which the trader is subject, and the trader does not process this data for any other purpose".

What is encompassed by the term "provides or undertakes to provide personal data" abovementioned? As highlighted by the Article 29 Working Party, ${ }^{10}$ the GDPR contains such term "data provided" in Article 20 (1) of the GDPR - "right to data portability". In its Guidelines, the term is broadly interpreted as encompassing data actively and knowingly provided by the data subject (for example, mailing address, user name, age, etc.) and observed data "provided" by the data subject by virtue of the use of the service or device (for example, a person's search history, traffic data and location data). However, there is no reason to dismiss a more restrictive interpretation of the term. ${ }^{11 / 12 / 13}$

the provision of online services to data subjects, adopted on 9 April 2019 (version for public consultation), available on: https://edpb.europa.eu/sites/edpb/files/consultation/edpb_draft_guidelines-art_6-1-bfinal_public_consultation_version_en.pdf.

${ }^{8}$ Note that DCD excludes certain categories of data from its scope in relation with the purpose of the processing: "the personal data provided by the consumer are exclusively processed by the trader for the purpose of supplying the digital content or digital service in accordance with the Directive or for allowing the trader to comply with legal requirements to which the trader is subject, and the trader does not process those data for any other purpose", Article 3(1) DCD.

${ }^{9}$ See recital 24 of the DCD: "For example, this Directive should apply where the consumer opens a social media account and provides a name and email address that are used for purposes other than solely supplying the digital content or digital service, or other than complying with legal requirements. It should equally apply where the consumer gives consent for any material that constitutes personal data, such as photographs or posts that the consumer uploads, to be processed by the trader for marketing purposes (...)".

${ }^{10}$ Article 29 Working Party Guidelines on the right to data portability, adopted on 13 December 2016, last revised and adopted on 5 April 2017, available on: https://ec.europa.eu/newsroom/Article29/ item-detail.cfm?item_id=611233.

${ }^{11}$ An adverse effect of such an interpretation could be that suppliers would avoid asking for the data to be directly provided but rather collect and process the same data passively provided by the consumers, in order to avoid being subject to the DCD.

${ }^{12}$ In any case, and according to Article 13(1)(c) of the GDPR, the purposes for which the data are processed must be provided to the data subjects, irrespective of whether the data was provided by them or not.

${ }^{13}$ See recital 25 of the DCD: "This Directive should also not apply to situations where the trader only collects metadata, such as information concerning the consumer's device or browsing history, except where this situation is considered to be a contract under national law. It should also not apply to situations where the consumer, without having concluded a contract with the trader, is exposed to advertisements exclusively in order to gain access to digital content or a digital service. However, Member States should remain free to extend the application of this Directive to such situations 
If personal data is depicted as counter-performance, companies can extensively process it as long as it is in exchange of a digital content or service. Alternatively, can this statement be too wide in scope and in need of clear boundaries set by data protection law?

Article 3 of the DCD alludes to the GDPR and states that in case of conflict between these two legal instruments, the GDPR should prevail. ${ }^{14}$ This is a clear indication of the particularity of personal data as counter-performance, as companies supplying this type of content or services in exchange for personal data will have to act in compliance with data protection legal requirements, i.e., process personal data lawfully. As such, companies will always need to rely on a legal ground when processing personal data provided in exchange of digital content or services. Nevertheless, can we consider that this legal ground will be the necessity for the performance of the contract entered into with the consumer, provided by Article 6(1)(b) of the GDPR?

The abovementioned EDPB's Guidelines may provide some insight into this, although they do not make any express reference to the DCD. Before addressing EDPB's Guidelines, it is important to analyze EDPS' Opinion 4/2017 ${ }^{15}$ from which stems the recommendation of avoiding the use of the notion of data as counterperformance. ${ }^{16}$

\section{The EDPS ${ }^{17}$ Opinion 4/2017}

The EDPS warns against any provisions stating "people can pay with their data the same way as they do with money. Fundamental rights such as the right to the protection of personal data cannot be not be reduced to simple consumer interests, and personal data cannot be considered as a mere commodity".

The EDPS acknowledges that the scope of the DCD has the objective to cover services generally considered as "free", which tend to be based on "an economic model where personal data are collected by the providers in order to create value from the data processed". However, personal data is not comparable to a price, as it is related to the fundamental right to the protection of personal data, as enshrined in Article 8 of the Charter of

or to otherwise regulate such situations, which are excluded from the scope of this Directive".

${ }^{14}$ See recital 37 of the DCD: "The pursuit of activities falling within the scope of this Directive could involve the processing of personal data. Union law provides a comprehensive framework on the protection of personal data. In particular, this Directive is without prejudice to Regulation (EU) 2016/679 and Directive 2002/58/EC of the European Parliament and of the Council. That framework applies to any personal data processed in connection with the contracts covered by this Directive. Consequently, personal data should only be collected or otherwise processed in accordance with Regulation (EU) 2016/679 and Directive 2002/58/EC. In the event of a conflict between this Directive and Union law on the protection of personal data, the latter should prevail."

${ }^{15}$ EDPS, Opinion 4/2017 on the Proposal for a Directive on certain aspects concerning contracts for the supply of digital content, 14.03.2017, available on: https://edps.europa.eu/sites/edp/files/ publication/17-03-14_opinion_digital_content_en.pdf.

${ }^{16}$ As stated by the EDPS, "in the EU, personal information cannot be conceived as a mere economic asset. According to the case law of the European Court of Human Rights, the processing of personal data requires protection to ensure a person's enjoyment of the right to respect for private life and freedom of expression and association. Furthermore, Article 8 of the EU Charter and Article 16 of the Treaty on the Functioning of the European Union (TFEU) have specifically enshrined the right to the protection of personal data. In consequence, the 2016 General Data Protection Regulation contains specific safeguards that could help remedy market imbalances in the digital sector", Opinion 8/2016 on coherent enforcement of fundamental rights in the digital age of big data, 23.09.2016, available on: https://edps.europa.eu/sites/edp/files/publication/16-09-23_bigdata_opinion_en.pdf. ${ }^{17}$ The EDPS is the EU's independent data protection authority. Bilateral and international cooperation with data protection authorities is part of EDPS activities particularly within the EDPB. Besides its role as full member of the EDPB, the EDPS also provides an independent secretariat for the EDPB. 
Fundamental Right of the EU and Article 16 of the TFEU, and further specified in secondary EU legislation - the GDPR and the e-Privacy Directive (to be replaced by the e-Privacy Regulation).

As such, the EDPS quite drastically states that; "there might well be a market for personal data, just like there is, tragically, a market for live human organs, but that does not mean that we can or should give that market the blessing of legislation". Whilst the comparison seems at first glance quite disproportionate, there is no doubt legal reasoning in avoiding the monetization of a fundamental right, as if it were nothing more than a commercial transaction. ${ }^{18}$

It is important to note that the EDPS' Opinion refers to the DCD in its version as a proposal and not as official text. In its final version, the DCD states that " (...) the protection of personal data is a fundamental right and that therefore personal data cannot be considered as a commodity". ${ }^{19}$ Furthermore, there are noticeable differences between paying a price with money and giving data as a counter-performance, as stated by the EDPS: " [w] bile the consumer is aware of what he is giving when he pays with money, the same cannot be said about data. Standard contractual terms and privacy policies do not make it easy for the consumer to understand what is precisely made with the data collected about him/her". As such, at the forefront, individuals cannot evaluate the value that will be created with their data.

This can be observed from the providers standpoint of view, as the obligation of restitution is, in principle, straightforward when a price is paid, but far more complex when data is exchanged. ${ }^{20}$

\section{The EDPB's Guidelines 2/2019 21}

The EDPB holds that Article 6(1)(b) of the GDPR may only be used as a legal basis where the processing is "objectively necessary for the performance of a contract with a data subject" or "objectively necessary in order to take pre-contractual steps at the request of a data subject". ${ }^{22}$ The EDPB further explains that "what is necessary for the performance of a contract is not simply an assessment of what is permitted by or written into the terms of the contract" and that "merely referencing or mentioning data processing in a contract is not enough to bring the processing in question within the scope of Article 6(1)(b)". The EDPB also mentions that although contracts for digital services can have express terms which impose additional conditions relating to "advertising, payments or cookies, amongst other things" this cannot

\footnotetext{
${ }^{18}$ Which does not dismiss recital 4 of the GDPR which very clearly states that " $[t]$ he right to the protection of personal data is not an absolute right; it must be considered in relation to its function in society and be balanced against other fundamental rights, in accordance with the principle of proportionality".

${ }^{19}$ See recital 24 of the DCD.

${ }^{20}$ There is notable difficulty in evaluating and determining the value of personal data, as well as in determining and giving compensation for the value gained by the supplier with the contract.

${ }^{21}$ Currently awaiting release of the public consultation results, which ended on the $24^{\text {th }}$ of May, available on: https://edpb.europa.eu/our-work-tools/public-consultations/2019/guidelines-22019processing-personal-data-under-Article-61b_en.

${ }^{22}$ The concept of what is 'necessary for the performance of a contract' is not a simple assessment of what is permitted by or written into the terms of a contract. The concept of necessity has an independent meaning in European Union law, which must reflect the objectives of data protection law, namely meaning that necessity is largely fact-based and must be considered in light of the specific circumstances (i.e. the context) of the processing and the purpose it aims to achieve (Huber Case; C-524/06). As such and according to the EDPB's Guidelines 2/2019: "Where controllers cannot demonstrate that (a) a contract exists, (b) the contract is valid pursuant to applicable national contract laws, and (c) that the processing is objectively necessary for the performance of the contract, the controller should consider another legal basis for processing."
} 
lead to "artificially expand the categories of personal data or types of processing operation that the controller needs to carry out for the performance of the contract within the meaning of Article 6(1)(b)".

As highlighted by Article 29 Working Party ${ }^{23}$ in relation to Directive 95/46 on the protection of individuals with regard to the processing of personal data and on the free movement of such data - repealed by the $\mathrm{GDPR}^{24}$ - the provision must be interpreted strictly and not extend to situations where the processing is not genuinely necessary for the performance of a contract.

As such, the number of cases in which the contract will be a lawful basis for the processing of personal data for the supply of digital content or services will be sparse. This is supplemented by the exceptions stated in Article 3 of the DCD, as personal data provided by the consumer exclusively processed by the trader for delivering the digital content falls outside the scope of DCD.

In addition, if the trader wants to build a profile of his/her customer and has mentioned this in the contract, this mere indication does not make this specific processing "necessary for the performance of the contract" 25 in light of data protection law and the trader will need to rely on a different legal basis to conduct the profiling activity.

As such, the mere fact that the purposes of the processing are covered by contractual clauses drafted by the trader will not automatically mean that the processing is necessary for the performance of the contract. In this regard, processing for "service improvement" or "fraud prevention" are likely to go beyond what is objectively necessary for the performance of a contract. The EDPB however acknowledges "processing for personalisation of content [...] may constitute an essential or expected element of certain online services, and therefore may be regarded as necessary for the performance of the contract with the service user in some cases". Yet, "where personalisation of content is not objectively necessary for the purpose of the underlying contract, for example, where personalised content delivery is intended to increase user engagement with a service but is not an integral part of using the service, data controllers should consider an alternative basis". ${ }^{26}$

The EDPB further refers another important distinction to be made by traders when providing online services: "between entering into a contract and giving consent within the meaning of Article 6(1)(a), as these concepts are not the same and have different implications for data subject's rights and expectations".

In addition, in accordance with the rules under the GDPR, the processing of special categories of personal data cannot be legitimized by the "necessity for the performance of a contract". Therefore, traders will likely need to seek explicit consent in accordance with the GDPR conditions when processing such data [provided none of the other exceptions under Article 9(2) applies].

It is also important to understand if traders can rely on Article 6(1)(b) as the legal basis for processing not just for the initial version of an online service, but also for its subsequent versions or updates, providing, of course, that all the criteria in Article 6(1) (b) are still fulfilled. ${ }^{27}$

\footnotetext{
${ }^{23}$ See Article 29 Working Party Opinion 06/2014 on the notion of legitimate interests of the data controller under Article 7 of Directive 95/46/EC, adopted on the 9.4.2014.

${ }^{24}$ Despite the Directive no longer being in force, there are strong similarities between the GDPR and the Directive regarding legal bases. For the comparison, see the Directive's Article 7 (b): “Member States shall provide that personal data may be processed only if processing is necessary for the performance of a contract to which the data subject is party or in order to take steps at the request of the data subject prior to entering into a contract".

${ }^{25}$ As exemplified in the EDPB's Guidelines 2/2019, page 9.

${ }^{26}$ See EDPB's Guidelines 2/2019, pages $13-14$.

${ }^{27}$ Note that service improvements are inherently necessary to keep services reasonably safe and
} 
In sum, the fact that the processing of personal data can be counter-performance received in exchange of the supply of digital content or services is not connected to the choice of the legal ground for the processing which is subject to GDPR requirements, ${ }^{28}$ as further explained in the next chapter. As such, a trader acting as a data controller may never assume that the signature of a contract corresponds to a consent in the sense of Article 6(1)(a) of the GDPR.

\section{Legal grounds for processing personal data}

In a digital environment and considering the rise of the value of personal data as an asset, one of the most important issues to address by a data controller is the identification of the appropriate legal basis for the envisaged processing operations, before the processing commences.

In the present case, the legal basis for the processing of data will most likely be the consent of data subjects..$^{29 / 30 / 31 / 32}$ Linked with this, and concerning the freedom to withdraw one's consent, ${ }^{33}$ note that the value generated by the supplier with the personal data of the consumer will, in general, have been created before the withdrawal of consent. As such, the lawfulness of the processing performed before the withdrawal will not be affected by the withdrawal. ${ }^{34}$ In this context, it is also important to verify if there is no imbalance between the data controller and the data subjects, as it deems consent invalid in such cases. A valid consent will depend of the positions of the parties and the existence of information asymmetries.

secure. As such, they need to be updated from time to time (e.g. via security patches) to ensure that users get the service they contracted for in a reasonably safe and secure fashion.

${ }^{28}$ See recital 38 of the DCD: "This Directive should not regulate the conditions for the lawfulprocessing of personal data, as this question is regulated, in particular, by Regulation (EU) 2016/679. As a consequence, any processing of personal data in connection with a contract falling within the scope of this Directive is lawful only if it is in conformity with the provisions of Regulation (EU) 2016/679 relating to the legal grounds for the processing of personal data."

${ }^{29}$ The Proposal for an e-Privacy Regulation also provides that the conditions of the GDPR must apply regarding the validity of consent. Therefore, all conditions around consent introduced by the GDPR apply to the processing of data in the context of the provision of digital content. One of the conditions regarding consent is transparency of the processing, which depends on the information given to the consumer regarding the processing. Yet it is (very) rare that the supplier explicitly states that the data will be used as counter-performance.

${ }^{30}$ In the EDPS' preliminary Opinion 5/2016 on the review of the e-Privacy Directive, dated 22.6.2016, it recommended that "the new provisions on e-Privacy should provide that no one shall be denied access to any information society services (whether these services are remunerated or not) on grounds that he or she has not given his or her consent under Article 5(3)", available on: https://edps.europa.eu/sites/edp/files/ publication/16-07-22_opinion_eprivacy_en.pdf.

${ }^{31}$ Article 29 Working Party Opinion 15/2011 on the definition of consent, dated 13.07.2011, (WP 187): "The choice of the most appropriate legal ground is not always obvious (...). A data controller using Article 7(b) as a legal ground in the context of the conclusion of a contract cannot extend it to justify the processing of data going beyond what is necessary: he will need to legitimize the extra processing with a specific consent to which the requirements of Article 7(a) will apply. This shows the need for granularity in contract terms. In practice, it means that it can be necessary to have consent as an additional condition for some part of the processing. Either the processing is necessary to perform a contract, or (free) consent must be obtained', available on: https://ec.europa.eu/justice/Article-29/ documentation/opinionrecommendation/files/2011/wp187_en.pdf.

${ }^{32}$ See recital 24 of the DCD: "The personal data could be provided to the trader either at the time when the contract is concluded or at a later time, such as when the consumer gives consent for the trader to use any personal data that the consumer might upload or create with the use of the digital content or digital service".

${ }^{33}$ As data subjects can agree to the processing of their personal data, which is not equivalent to trading away their fundamental rights.

${ }^{34}$ See Article 7(3) of the GDPR. 
A very relevant aspect, which the DCD does not cover, is the consequences for the contracts covered by it in the event that the consumer withdraws the consent for the processing of the consumer's personal data. On the contrary, the DCD states that "ss]uch consequences should remain a matter for national law" 35 thus avoiding the contractual harmonization envisaged in its making.

Processing may also take place when it is necessary for the purposes of the legitimate interests pursued by the data controller (or by a third party). In this case, a balancing exercise must be performed between the legitimate interest pursued by the controller and the fundamental rights and freedoms of the data subject. Such balance relating to the legitimate interest of the data controller (or third parties) has to be pursued on a case-by-case basis. Note that the European Court of Justice stated that the data subject's fundamental rights override, as a rule, the economic interests of an operator. ${ }^{36}$ Moreover, as stated by the EDPS; "the legal basis of consent is more protective for the consumer than the use of legitimate interest'. ${ }^{37}$

In addition, processing may also take place when it is necessary for compliance with a legal obligation to which the controller is subject. In such case, the purpose of ensuring that the digital content is in conformity with the legal requirements could be considered as processing performed on this legal ground.

\section{Lack of conformity of the digital content or service in light of GDPR requirements}

According to the DCD, facts leading to a lack of compliance with requirements provided for by the GDPR - including principles such as the requirements for data minimization, data protection by design and data protection by default - may be considered as a lack of conformity of the digital content / digital service with subjective or objective requirements, depending on the circumstances of the case. ${ }^{38}$

The DCD offers as an example the case in which a trader assumes a contractual obligation linked to his GDPR obligations, thus becoming part of the subjective requirements for conformity. For an example relating to the objective requirements for conformity, one may attend to the non-compliance by the trader of his GDPR obligations, thus rendering the digital content or service unfit for its intended purpose ${ }^{3940}$ or for the purpose one can reasonably expect. ${ }^{41}$ As such, where the facts

\footnotetext{
${ }^{35}$ See Recital 40 of the DCD.

${ }^{36}$ Judgment CJEU, Google Spain, C-131/12, 13.5.2014.

${ }^{37}$ The EDPS successfully argues in its Opinion 4/2017 that "the right of withdrawal of consent (...) is more protective than the right to object; the right to data portability (...) does not apply when the processing is based on legitimate interests, the conditions to obtain consent are more protective for the individuals since it requires an active action from his/ her side".

${ }^{38}$ See recital 48 of the DCD.

${ }^{39}$ See recital 48 of the DCD.

${ }^{40}$ See recital 48 of the DCD: "This would be the case, for example, if the trader of data encryption software fails to implement appropriate measures as required by Regulation (EU) 2016/679 to ensure that by design personal data are not disclosed to unauthorised recipients, thus rendering the encryption software unfit for its intended purpose which is the secure transferring of data by the consumer to their intended recipient".

${ }^{41}$ See recital 48 of the DCD: "For instance, if the trader of an online shopping application fails to take the measures provided for in Regulation (EU) 2016/679 for the security of processing of the consumer's personal data and as a result the consumer's credit card information is exposed to malware or spyware, that failure could also constitute a lack of conformity of the digital content or digital service within the meaning of this Directive, as the consumer would reasonably expect that an application of this type would normally possess features preventing the disclosure of payment details".
} 
leading to non-compliance with GDPR requirements constitute a lack of conformity of the digital content or service with subjective or objective requirements, the data subject should be entitled to the remedies for the lack of conformity provided for by the DCD.

Even more revealing is the level of lack of conformity required to trigger termination of contract. In cases where the digital content or service is supplied in exchange for a price, the data subject will be able to terminate the contract only if the lack of conformity is not minor. However, if the digital content or service is supplied in exchange for personal data, the data subject will be entitled to terminate the contract also in cases where the lack of conformity is minor. The reason for this lies in the fact that the remedy of price reduction is not available to the data subject in the latter situation. In cases where the consumer pays a price and provides personal data, the consumer should be entitled to all available remedies in the event of a lack of conformity. In particular, provided all other conditions are met, the consumer should be entitled to have the digital content or digital service brought into conformity, to have the price reduced in relation to the money paid for the digital content or digital service or to have the contract terminated. ${ }^{42}$

\section{Conclusion}

Although the DCD aims to protect consumers, it legitimizes a practice: treating personal data as counter-performance. Can this mean that the same person is now more protected as a consumer and less protected as a data subject? Or, alternatively, do the provisions of the DCD relating to the lack of conformity of the digital content or service in light of GDPR requirements, analyzed above, ${ }^{43}$ bridge the gap between these two "positions"?

The EDPS recommended the term "data as a counter-performance" be avoided. Nevertheless, would that suffice, and would it have any effect on existing practices? ${ }^{44}$

Bearing what has been analyzed, it is safe to say that we are not facing colliding worlds, albeit there is much to be harmonized between the DCD and the GDPR, in which the Court of Justice will have a fundamental role, and between the DCD and national civil laws. In any case, in no scenario should the DCD change the balance pursued by the GDPR regarding the processing of personal data taking place in the digital market.

\footnotetext{
${ }^{42}$ See recital 67 of the DCD: "In cases where the consumer pays a price and provides personal data, the consumer should be entitled to all available remedies in the event of a lack of conformity. In particular, provided all other conditions are met, the consumer should be entitled to have the digital content or digital service brought into conformity, to have the price reduced in relation to the money paid for the digital content or digital service or to have the contract terminated'.

${ }^{43}$ See recital 48 of the DCD: "Facts leading to a lack of compliance with requirements provided for by Regulation (EU) 2016/679, including core principles such as the requirements for data minimisation, data protection by design and data protection by default, may, depending on the circumstances of the case, also be considered to constitute a lack of conformity of the digital content or digital service with subjective or objective requirements for conformity provided for in this Directive".

${ }^{44}$ The EDPS offered another interesting solution which (although not adopted) was a valuable alternative in avoiding the terminology: "use similar terms to the GDPR (referring to the offering of goods and services irrespective of whether a payment is required) in order to define the scope of the Proposal, without making reference to data used as counter-performance".
} 